\title{
The Novel Object and Unusual Name (NOUN) Database: A collection of novel images for use in experimental research
}

\author{
Jessica S. Horst ${ }^{1}$ - Michael C. Hout ${ }^{2}$
}

Published online: 30 September 2015

(C) Psychonomic Society, Inc. 2015

\begin{abstract}
Many experimental research designs require images of novel objects. Here we introduce the Novel Object and Unusual Name (NOUN) Database. This database contains 64 primary novel object images and additional novel exemplars for ten basic- and nine global-level object categories. The objects' novelty was confirmed by both self-report and a lack of consensus on questions that required participants to name and identify the objects. We also found that object novelty correlated with qualifying naming responses pertaining to the objects' colors. The results from a similarity sorting task (and a subsequent multidimensional scaling analysis on the similarity ratings) demonstrated that the objects are complex and distinct entities that vary along several featural dimensions beyond simply shape and color. A final experiment confirmed that additional item exemplars comprised both suband superordinate categories. These images may be useful in a variety of settings, particularly for developmental psychology and other research in the language, categorization, perception, visual memory, and related domains.
\end{abstract}

Keywords Novel objects $\cdot$ Unnameable stimuli $\cdot$ Picture database $\cdot$ Categorization $\cdot$ Novelty ratings $\cdot$ Word learning

Electronic supplementary material The online version of this article (doi:10.3758/s13428-015-0647-3) contains supplementary material, which is available to authorized users.

Jessica S. Horst

jessica@ sussex.ac.uk

1 School of Psychology, University of Sussex, Pevensey 1 Building, Falmer, Brighton, East Sussex BN1 9QH, UK

2 Department of Psychology, New Mexico State University, Las Cruces, NM, USA
Many psychological experiments involve word learning tasks, in which participants - both adults and children - are taught names for objects either explicitly - for instance, through the use of social cues and ostensive feedback (Gauthier \& Tarr, 1997; Horst \& Samuelson, 2008)—or implicitly across several encounters (e.g., Axelsson \& Horst, 2014; Yu \& Smith, 2007). In studies such as these, novelty is critical for ensuring that researchers are testing learning that has occurred as a function of the experimental manipulation and not merely tapping into knowledge acquired prior to the experiment (Ard \& Beverly, 2004; Bornstein \& Mash, 2010). In addition, novelty is often critical for categorization studies in which participants must learn to extrapolate information from one category exemplar and generalize or apply that information to new exemplars (e.g., Homa, Hout, Milliken, \& Milliken, 2011; J.D. Smith \& Minda, 2002).

Previous research demonstrates that novelty (or the lack thereof) can have a profound effect on subsequent learning. For example, after toddlers explore novel objects for 1-2 min, they are significantly less likely to associate novel names with those objects than with still-novel objects (Horst, Samuelson, Kucker, \& McMurray, 2011; see also Kucker \& Samuelson, 2012). Thus, even brief prior experience with stimuli can change subsequent behavior on critical test trials. Likewise, gradual, prior experience with stimuli can also influence subsequent behavior, such as looking times during the learning phase of an object-examination categorization task (Bornstein $\&$ Mash, 2010). It is therefore ideal that visual stimuli not have been seen before, in order to ensure that any inferences made regarding learning were not actually due to participants' exposure to the items prior to the experiment. For many experimental designs it is important that objects also be easy to distinguish from each other (e.g., Twomey, Ranson, \& Horst, 2014; Yu \& Smith, 2007); however, for other designs 
it can be useful to have objects that are somewhat similar (e.g., Homa et al., 2011; Hout \& Goldinger, 2015).

There are existing databases of known, familiar, real-world objects (e.g., Brady, Konkle, Alvarez, \& Oliva, 2008; DanGlauser \& Scherer, 2011; Hout, Goldinger, \& Brady, 2014; Konkle, Brady, Alvarez, \& Oliva, 2010; Migo, Montaldi, \& Mayes, 2013) and human faces (e.g., Ebner, Riediger, \& Lindenberger, 2010; Matheson \& McMullen, 2011), as well as databases of related items uniquely optimal for use in categorization studies (e.g., Gauthier \& Tarr, 1997; Marchewka, Żurawski, Jednoróg, \& Grabowska, 2014). However, researchers investigating memory for objects and object names critically need a database of novel objects for use in such experiments, where it is critical that participants have no a priori knowledge of the stimuli and that the objects not already be associated with specific names. The NOUN database is such a collection of novel object images.

\section{Why use the NOUN Database?}

The NOUN Database offers several advantages for researchers requiring images of unusual objects. First, the images in the NOUN Database depict multipart, multicolored, real three-dimensional (3-D) objects, as opposed to simple geometric shape configurations (e.g., L.B. Smith \& Yu, 2008; Wu, Gopnik, Richardson, \& Kirkham, 2011) or seemingly animate objects (e.g., Gauthier \& Tarr, 1997; Mather, Schafer, \& Houston-Price, 2011; Rakison \& Poulin-Dubois, 2002). As such, these stimuli are ideal for researchers who need images of naturalistic, complex novel objects to present against images of real 3-D objects that are already familiar to participants (e.g., familiar distractors or known competitors). Indeed, complex novel objects are often presented against known objects - for example, in language research (e.g., Axelsson \& Horst, 2014; Giezen, Escudero, \& Baker, in press; Mather \& Plunkett, 2009; Warren \& Duff, 2014; Zosh, Brinster, \& Halberda, 2013). In such cases, it is vital that the novel stimuli be just as "credible" as the familiar, known objects, which requires similar shading, colors, textures, and complexity. The stimuli in the NOUN Database have such properties because they are images of real objects (e.g., they are not "impossible" objects that might be created from a software package).

Second, researchers frequently choose their stimuli on the basis of their own intuitive judgments of novelty and similarity (Migo et al., 2013). This practice is especially prevalent in developmental psychology, where researchers make assumptions about objects that are unlikely to be familiar to young children without prior confirmation (but see Horst \& Samuelson, 2008, for a quick confirmation method). This can be problematic, because children may be implicitly learning about the object categories although they have not yet heard the category names. In experiments requiring images of novel objects, this problem can be avoided by using the preexisting NOUN Database; the novelty and similarity ratings we present can inform researchers' decisions on which stimuli to use, depending on their research questions. Specifically, these ratings can be used to ensure that a subset of stimuli are equally novel and not already associated with a specific name, as well as to vary the novelty or similarity across items.

Relatedly, using an existing database facilitates comparison across experiments, which can be especially helpful when different experiments address unique but related research questions, or when one wants to compare related effects. For example, young children generalize names for nonsolid substances to other substances made of the same material (Imai \& Gentner, 1997; Soja, Carey, \& Spelke, 1992), but only when the substances share both the same material and color-if the stimuli only share the same material, this effect disappears (Samuelson \& Horst, 2007). Similarly, adults are faster to repeat nonwords from high-density lexical neighborhoods than those from low-density neighborhoods (Vitevitch \& Luce, 1998), but this effect disappears with different stimuli-for example, when the leading and trailing silences in the audio files are removed to equate stimulus duration (Lipinski \& Gupta, 2005). The use of existing stimuli is also consistent with the recent push in the psychology research community to share resources and to facilitate replicability (for a lengthier discussion, see Asendorpf et al., 2013).

Finally, using an existing set of stimuli saves time and reduces research expenses because data collection on the substantive experiment can begin quickly, without the need for additional preliminary experiments that ensure that the stimuli are in fact novel and unlikely to already be associated with a particular name. The present Experiment 1 is effectively a preliminary experiment conducted on behalf of researchers who wish to use the NOUN Database. This is valuable, because obtaining and selecting experimental stimuli is often a highly time-consuming phase of the research process (DanGlauser \& Scherer, 2011; see also Umla-Runge, Zimmer, Fu, \& Wang, 2012). Even using 3-D rendering software can take time to learn and can be expensive. Moreover, using an existing database utilized by multiple researchers may expedite the ethical approval process for new studies. Taken together, these time-saving aspects make the NOUN Database particularly useful to students who must conduct research projects quickly with a strict deadline, as well as early-career researchers who may especially benefit from a reduced time to publication (see A. K. Smith et al., 2011, for a related argument).

Although one advantage of the NOUN Database is its ability to save valuable time and money, researchers using the database may choose to conduct their own preliminary experiments to ensure that the stimuli that will be used in their main 
experiment are in fact novel to their participant pool. Researchers can also use the images in the NOUN Database as a supplement to their own stimuli, which offers even greater experimental flexibility. Note that a second advantage of the NOUN Database is its size: It includes 64 items, which is many times more than is often required for most studies using images of novel objects (e.g., two novel objects, Bion, Borovsky, \& Fernald, 2013; three novel objects, Rost \& McMurray, 2009; one novel object, Werker, Cohen, Lloyd, Casasola, \& Stager, 1998).

\section{The present experiments}

The original NOUN Database included 45 images of novel objects (Horst, 2009). Each of these objects was distinct, and together the objects included a variety of shapes, colors, and materials. We have expanded the NOUN Database to include a total of 64 objects. In Experiment 1, adult participants judged the novelty of each object. Participants were asked whether they were familiar with each object, then what they would call each object, and finally what they thought each object really was. In Experiment 2, we used a multidimensional scaling (MDS) task to examine the extent to which the objects were complex and distinct. Specifically, we wanted to ensure that the objects were complex enough that participants were not only appreciating one or two featural dimensions (e.g., color and shape) when considering the objects. Finally, in Experiment 3, we repeated the MDS task with multiple exemplars from ten of the object categories, to determine the relationships between the various novel object categories, enabling us to make recommendations as to which subcategories belong to the same global-level categories.

This database was originally created for use in word learning experiments, primarily with children. However, researchers may also require novel objects when investigating categorization (e.g., Twomey et al., 2014), (visual) short-term memory (e.g., Kwon, Luck, \& Oakes, 2014), and long-term visual memory (e.g., Hout \& Goldinger, 2010, 2012). This newly-developed set of photographs is freely available to the scientific community from the authors for noncommercial use.

\section{Experiment 1}

Our goal with this first experiment was to test whether the novel objects in the NOUN Database are, in fact, generally novel across participants. Novelty is on a continuum (Horst, 2013) and could imply that a stimulus has never been encountered before or has been previously encountered but never associated with a particular name. Due to this plurality of possible definitions, we examined the novelty of the NOUN stimuli using multiple tasks that would each tap into a different (but related) aspect of what it means for something to be novel. First, we simply asked participants whether they had seen each object before. One might argue that this is the ultimate test of novelty. Next, we asked participants what they would call each object. It may be that an object has never been seen before but is highly reminiscent of a known object. The name question allowed us to examine this conceptualization of novelty. Finally, we asked participants what they thought each object really was. This question was included (in addition to the name question, with which it may seem partially redundant) because it is possible to name something on the basis of its appearance but to know that it is not really from a particular category. For example, someone might know that something looks like a clothespin but is really art (Landau, Smith, \& Jones, 1998), looks like a bowtie but is really pasta, or looks like a jiggy but is really a zimbo (Gentner, 1978).

\section{Method}

Participants Undergraduate students participated for course credit. Each participant gave written informed consent and then completed all three experiments. Before data collection began, the authors agreed on a target sample size of $n=30$, based on previous studies of adult ratings of stimuli for use in experiments with children (e.g., Horst \& Twomey, 2013; Samuelson \& Smith, 1999, 2005). Participants signed up to participate using an online sign-up system. Initially, 47 students signed up to participate before sign-ups were closed. Six students canceled and another six students failed to attend their scheduled sessions. The remaining 35 students showed up to participate. The data from three participants were excluded from all analyses because of equipment failure $(n=1)$ or failure to follow the instructions $(n=2)$. This resulted in a final sample of 32 participants ( 20 women, 12 men). Neither author analyzed any data until after all 32 participants had completed the study.

Materials Photographs were taken of real, 3-D objects against a white background. These raw images were then imported into Adobe Photoshop, where the backgrounds were deleted to create the cleanest image possible. Images were saved as Jpegs at 300 dots per inch (DPI). They are also available at 600 DPI. Images of five of the objects are available only at a lower resolution (200-500 pixels/in.) because these objects were no longer available for photographing; for example, one object was a ceramic bookend that shattered shortly after the original photograph was taken. These objects have remained in the database for continuity because they were present in the original database. Each item in the database is assigned a unique, four-digit ID number (cf. catalog number), to facilitate communication between researchers. The sequence of the ID numbers was completely random. 
Procedure and design Participants completed the experiment on lab computers either individually or in pairs (on separate machines on different sides of the lab). Before each task, written instructions were displayed on the screen. Participants first completed the novelty questions. Objects were displayed individually in the center of the screen in a random order. Below each object, the question "Have you seen one of these before? (enter $y / n$ )" was written. Participants responded by pressing the $\mathrm{Y}$ or the $\mathrm{N}$ key. After each response the next object was displayed, until participants had responded to each of the 64 novel objects.

Next, participants answered the question "What would you call this object?" for each object. This question was asked in order to determine the degree of consensus among participants as to what to call an object; we refer to this item as the "name question." Again, objects were displayed individually in the center of the screen in a random order. Below each object was a black text box in which participants could freely type their responses, which appeared in white font. Participants were instructed to type "XXX" if they wanted to skip an object (although the computer also accepted blank responses). ${ }^{1}$ Participants pressed the return key to advance to the next object. After each response the next object was displayed, until participants had responded to each of the 64 novel objects.

Finally, participants answered the question "What do you really think this is?" for each object. This question was asked in order to determine the consensus regarding what each object really was; we refer to this item as the "identity question." Objects and a free-response text box were displayed as in the previous task. Participants were instructed to type "XXX" if they did not have an answer (although the computer again accepted blank responses). ${ }^{2}$ Participants pressed the return key to advance to the next object. After each response the next object was displayed, until participants had responded to each of the 64 novel objects. Objects were presented in a random order for each of the tasks.

Data were collected on a Dell computer. Each display was a 17-in. (43.18-cm) LCD monitor, with resolution set to 1,280 × 1,024 pixels and refresh rate of $60 \mathrm{~Hz}$. E-Prime (Version 2.0, Service Pack 1; Schneider, Eschman, \& Zuccolotto, 2002) was used to control stimulus presentation and collect responses.

\footnotetext{
${ }^{1}$ One participant entered "XXX" 11 times. This participant and four others left answers blank a total of 54 times $\left(M_{\text {nonresp }}=10.8, S D_{\text {nonresp }}=\right.$ 11.9 , range $=1-29$, for these five participants). In total, among all 32 participants, only $2 \%$ of the description questions were unanswered.

${ }^{2}$ In total, 26 of the 32 participants entered "XXX" or a blank response for at least one item $\left(M_{\text {nonresp }}=32.62, S D_{\text {nonresp }}=19.04\right.$, range $=1-64$, for these participants). In total, among all 32 participants, $21 \%$ of the identity questions were unanswered.
}

Coding The verbatim responses to the name and identity questions were corrected for typos (e.g., letter omission: "ornge" to "orange"; letter order: "bule stuff" to "blue stuff"; or wrong key on the keyboard: "squeexy toy" to "squeezy toy") and spelling errors (e.g., "marakah" to "maraca," "raidiator plug" to "radiator plug," "aunement" to "ornament"-in this case, the participant had typed "aunement: sorry bad spelling"). In most cases corrections were facilitated because other participants had provided correctly spelled responses (e.g., "ornament") for the same item. Circumlocution responses were coded as if the participant had used the noun he or she was describing: Examples include "help put on stubborn shoes," coded as "shoehorn"; "a toy to help children learn shapes: have to put 3-D objects through the holes," coded as "shape sorter"; and "a fluorescent multi-colored object, which you could find in a fish tank, mainly fluorescent pink, also is shaped like the Sydney Opera House," coded as "fish tank accessory (fluorescent pink and multicolored)" (here "fish tank accessory" was used over "Sydney Opera House" because it occurred first in the response).

In many cases participants qualified their responses. However, because some participants spontaneously qualified their statements and others did not, qualifiers were not included when determining consensus (percent agreement). For example, for Object 2033, "box," "orange box," "diamond box," "orange diamond box," and "orange crate box" were all coded as agreeing on "box." In the present study, consensus among the participants was more conservatively biased against our hypothesis that these are generally novel objects; therefore, we coded these responses as if they were not qualified when calculating percent agreement. For example, both "alien looking thing" and "alien" were coded as "alien" (which increased participant consensus). This is in line with Landau, Smith, and Jones (1998), who explained that object naming may be qualified, but the important content is the noun (which explains, among other things, why people refer to Philadelphia's 60-foot sculpture of a clothespin as a "clothespin," although it cannot possibly be used for that function).

Finally, for the consensus analyses, synonyms were collapsed, which increased participant agreement and was therefore again conservative against our hypothesis that it is difficult to agree on names for these items. Examples included "cylinder" and "tube," "maraca" and "shaker," and "racket" and "paddle." This influenced name consensus for 39 objects $\left(M_{\text {raw }}=35 \%, S D_{\text {raw }}=14 \% ; M_{\text {adjus }}=47 \%, S D_{\text {adjus }}=19 \%\right)$ and identity consensus for 33 objects $\left(M_{\text {raw }}=38 \%, S D_{\text {raw }}=17 \%\right.$; $M_{\text {adjus }}=49 \%, S D_{\text {adjus }}=20 \%$ ). Consensus scores were calculated out of the number of participants who provided a response for a given object. For example, 11 participants responded that Object 2010 was really a bone $(n=1)$ or a dog toy $(n=10)$, but only 21 participants provided a response for that object, yielding 52\% agreement. Again, this was a 
more conservative approach than taking the absolute agreement (e.g., 11 out of 32 would be $34 \%$ ).

\section{Results and discussion}

The objects in the NOUN Database are generally novel, as indicated by self-report: $M_{\text {novelty }}=69 \%, S D_{\text {novelty }}=19 \%$, range $=19 \%-97 \%$. This novelty was also reflected in the (lack of) consensus as to what to call the objects $(M=47 \%, S D=$ $19 \%$ ) and the (lack of) consensus as to what the objects really were $(M=49 \%, S D=20 \%)$. These rates are significantly less than the $85 \%$ agreement threshold set by Samuelson and Smith (1999), both $t \mathrm{~s}>14.20$ and $p \mathrm{~s}<.001$, two-tailed, both $d s>1.76$. Using Samuelson and Smith's (1999) threshold, participants agreed on what to call Objects 2002, 2003, 2032 , and 2059 , but only when we collapsed across synonyms. Note that we were particularly lenient in accepting synonyms for Object 2032 and included any names for vehicles or flying objects. Using the same threshold, participants agreed on what Objects 2032 and 2059 really were, but again only when we collapsed across synonyms.

Novelty scores were negatively correlated with both name consensus $(r=-.290, p=.02,95 \%$ confidence interval $[\mathrm{CI}]=$ -.048 to -.500$)$ and identity consensus $(r=-.465, p<.001$, $95 \% \mathrm{CI}=-.247$ to -.638 ). That is, the more novel the object, the less likely were participants to agree on the name or the identity of the object. If an object is familiar, it should be easier to have agreement on what it is and what to call it (especially when collapsed across synonyms, which we had done). Thus, these negative correlations provide additional evidence that the novelty scores are reliable.

When viewing the free responses during the consensus analyses, the use of qualifiers was staggering. We would be remiss if we did not disseminate these findings. In total, participants spontaneously provided 1,426 color and texture qualifiers in their statements, although each object was presented separately on a decontextualized background. For each object, the proportion of colors and proportion of textures (e.g., "spikey," "soft") for the name and identity questions were calculated as the number of qualifiers given the number of responses. Proportions of qualifiers were submitted to a qualifier type (color, texture) by trial type (name question, identity question) repeated measures analysis of variance (ANOVA). The ANOVA yielded a significant qualifier type by trial type interaction, $F(1,63)=159.19, p<.001, \eta_{\mathrm{p}}{ }^{2}=.72$ (see Fig. 1), indicating that participants were both significantly more likely to use color terms $(M=.27, S D=.15)$ than texture terms $(M=$ $.10, S D=.14)$ and significantly more likely to use qualifiers when asked what they would call something $(M=.33, S D=$ $.15)$ than when asked what something really is $(M=.04, S D=$ .07). Significant main effects of qualifier type, $F(1,63)=$ $88.30, p<.001, \eta_{\mathrm{p}}{ }^{2}=.58$, and trial type, $F(1,63)=617.72$, $p<.001, \eta_{\mathrm{p}}{ }^{2}=.91$, were also found. The greater use of

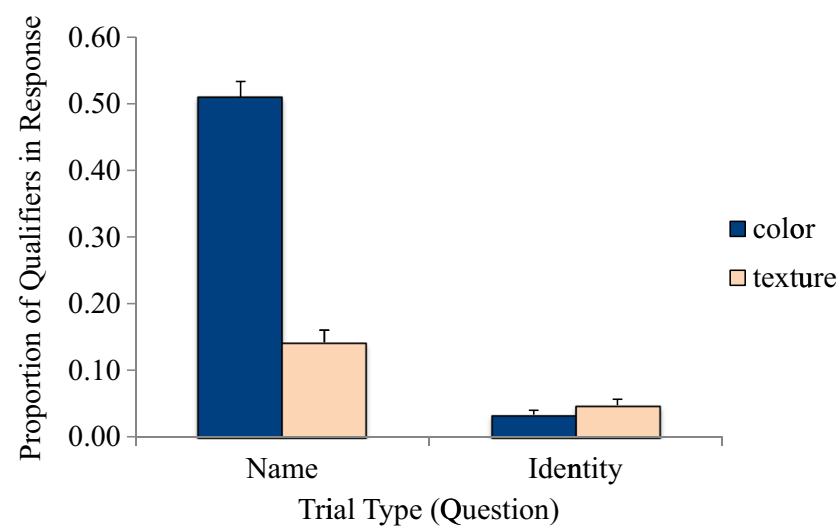

Fig. 1 Number of qualifiers as a function of trial type (question), from Experiment 1. Error bars represent one standard error

qualifiers on the name trials may reflect participants' uncertainty when asked what to name something novel. Indeed, the proportion of color qualifiers on the naming trials was significantly correlated with novelty, $r=.42, p=.0006,95 \% \mathrm{CI}=$ .189-.599. Thus, when participants did not know what something was they relied on color to refer to the object. This pattern of responding can also be seen if we compare the number of color qualifiers for self-reported novel versus known objects, $t(63)=7.93, p<.0001$, two-tailed, $d=1.14$

\section{Experiment 2}

In Experiment 2, our participants provided similarity ratings on the NOUN stimuli, which were subjected to an MDS analysis. It should be noted that MDS is not the only approach we could have adopted. There are a variety of models of similarity, and each has its own assumptions regarding the fundamental ways in which psychological similarity is represented by people, the ways in which similarity estimates are constructed, and so on (see Hahn, 2014, for a discussion). Spatial models of similarity represent objects as points in a hypothetical "psychological space," wherein the similarity of a pair of items is represented by their distance in space (with like items being located close to one another, and unlike items far away from one another; see Shepard, 1980, 1987). MDS techniques are specifically designed to "uncover" these psychological maps, and have even been successfully incorporated into sophisticated mathematical models of cognition, such as the generalized context model (GCM; Nosofsky, 1986).

An alternative approach to adopting a spatial model of similarity would be to examine similarity from the perspective of featural accounts, which assume that the basic representational units of similarity are not continuous, but are binary. Proponents of featural accounts point out that spatial models have some problematic assumptions, such as symmetry constraints (e.g., a Chihuahua may be rated as being more similar to a Labrador than a Labrador is to a Chihuahua) and the 
triangle inequality axiom (see Goldstone \& Son, 2012; Tversky, 1977). Statistical techniques such as additive clustering (e.g., Shepard \& Arabie, 1979) are typically adopted when the analyst assumes a featural account of similarity. More recent approaches have even extended these tools to accommodate continuous dimensions and discrete features using Bayesian statistics (see Navarro \& Griffiths, 2008; Navarro \& Lee, 2003, 2004).

Our primary reasons for adopting a spatial model of similarity relates to the novelty and real-world nature of the NOUN stimuli. With featural models, detailed predictions about similarity structure are sometimes difficult to make, because specific assumptions need to be made regarding the features that comprise the objects. The NOUN stimuli are complex, real-world objects, but their novelty makes it difficult to make any predictions regarding any features from which they may be comprised. Spatial models (implemented using MDS), however, are agnostic about these features. Moreover, upon inspection of the objects themselves, it seems more likely that the salient features would be continuous, rather than binary, making a spatial model more attractive to adopt. For instance, because many of the objects are brightly multicolored, a continuous color dimension seems more appropriate than a featural representation (e.g., an object may be entirely green, mostly green, partially green, and so on). Finally, we find spatial models appealing in general, because of their successful incorporation into exemplar models of cognition. Exemplar models, such as the GCM (Nosofsky, 1986), are adept at capturing human categorization and recognition behavior, and these are precisely the types of issues for which we hope future researchers will utilize the NOUN stimuli.

\section{Method}

Participants The same participants as in Experiment 1 took part in Experiment 2. This experiment took place immediately after Experiment 1.

Materials The same stimuli were used as in Experiment 1.

Procedure and design Participants were shown all 64 objects across 13 trials and provided similarity ratings using the spatial arrangement method (SpAM; Hout, Goldinger, \& Ferguson, 2013; see also Goldstone, 1994). On each trial, 20 different pictures were shown to the participant, arranged in four discrete rows (with five items per row), with random item placement. Participants were instructed to drag and drop the images in order to organize the space, such that the distance among the items would be proportional to each pair's similarity (with items closer in space denoting greater similarity). Participants were given as much time as they needed to arrange the space on each trial; typically, trials lasted between 2 and $5 \mathrm{~min}$. Once participants had finished arranging the items, they completed the trial by clicking the right mouse-button. To avoid accidental termination, participants were asked if the space was satisfactory (indicating responses via the keyboard) and were allowed to go back and continue the trial if desired.

The $x$ - and $y$-coordinates of each image was then recorded, and the Euclidean distance (in pixels) between each pair of stimuli was calculated (for 20 stimuli, there are 190 pairwise Euclidean distances). This procedure was performed repeatedly (over 13 trials), but with different images being presented together on each trial, so that all pairwise comparisons among the 64 total images were recorded. Thus, this provided a full similarity matrix comparing the ratings of each image to all of the other images (i.e., all 2,016 comparisons) for each participant. This took participants under an hour to complete; similar rating procedures have been used by other researchers (Goldstone, 1994; Hout, Papesh, \& Goldinger, 2012; Kriegeskorte \& Mur, 2012).

Stimulus selection We controlled the selection of images on each trial by employing a Steiner system (see Doyen, Hubaut, \& Vandensavel, 1978); these are mathematical tools that can be used to ensure that each item in a pool is paired with every other item (across subsets/trials) at least once. A Steiner system is often denoted $S(v, k, t)$, where $v$ is the total number of stimuli, $k$ is the number of items in each subset, and $t$ is the number of items that need to occur together. Thus, for us, $v, k$, and $t$ equaled 64 (total images), 20 (images per trial), and 2 (denoting pairwise comparisons), respectively. Simply put, the Steiner system provides a list of subsets (i.e., trials) identifying which items should be presented together on each trial. For some combinations of $v$ and $k$, a Steiner set may exist that does not repeat pairwise comparisons (i.e., each pair of items is shown together once and only once). For other combinations (including ours), some stimuli must be shown with others more than once. Because this leads to multiple observations per "cell," we simply took the average of the ratings for the pairs that were shown together more than once. Across participants, images were randomly assigned to numerical identifiers in the Steiner system, which ensured that each participant saw each pair of images together at least once, but that different people received different redundant pairings (see also Berman et al., 2014, for a similar use of multitrial implementations of the SpAM).

MDS analysis After the similarity matrices were compiled, we performed MDS on the pairwise Euclidean distances, using the PROXSCAL scaling algorithm (Busing, Commandeur, Heiser, Bandilla, \& Faulbaum, 1997). The data were entered into SPSS in the form of individual similarity matrices for each participant; averaging was performed by the PROXSCAL algorithm automatically. To determine the correct dimensionality of the space, we created scree plots, plotting the model's stress against the number of dimensions used 
in the space (see also Hout, Papesh, \& Goldinger, 2012). Stress functions vary across scaling algorithms (PROXSCAL uses "normalized raw stress"), but all are computed to measure the agreement between the estimated distances provided by the MDS output and the raw input proximities (lower stress values indicate a better model fit). Scree plots are often used to determine the ideal dimensionality of the data by identifying the point at which added dimensions fail to improve the model fit substantially (Jaworska \& Chupetlovska-Anastasova, 2009).

PROXSCAL offers several options for determining the starting configuration of the MDS space (prior to the iterative process of moving the points around in space to improve model fit). Specifically, there are Simplex, Torgerson, and multiple-random-starts options. The first two options use mathematical principles to determine the starting configuration, whereas the latter takes multiple attempts at scaling, using completely random configurations (the output with the lowest stress value is then reported). The benefit of the multiple-random-starts approach is that with deterministic starting configurations (like those used by Simplex and Torgerson algorithms), as the iterative process of moving the items in the space progresses, there is a risk that the solution will fall into a local (rather than a global) minimum with respect to stress. Because the process is repeated many times under a multiple-random-starts approach, that risk is reduced. We created scree plots using each of the options (for multiple random starts, we implemented 100 random starting configurations per dimensionality).

\section{Results and discussion}

Scree plots Scree plots for each of the starting configuration options are shown in Fig. 2. It is clear from these plots that the data comprised more than just two primary featural dimensions. Each space exhibits a remarkably similar shape, with stress values beginning to plateau at around four dimensions.
Configuration In order to choose the "best" solution to report, we correlated the interitem distance vectors across solutions derived from each of the three starting configurations (see Hout, Goldinger, \& Ferguson, 2013, and Goldstone, 1994, for similar approaches). This provides a metric to gauge the extent to which the arrangement of points in one MDS space "agrees" with the others (e.g., a pair of points that is located close together in one space should be close together in the others, and vice versa). Seeing that stress values were nearly identical across solutions (see Fig. 2), we then chose to report the configuration derived from the Torgerson starting configuration, because its organization correlated the most strongly with the other two (Pearson correlation coefficients with the Simplex and multiple-random-starts configurations were .72 and .75 , respectively, indicating a high degree of agreement across spaces). Below, we provide the scaling solution based on the Torgerson option in four-dimensional space. The results are shown in Figs. 3 and 4. In the figures, the objects are superimposed on the resulting MDS plot, such that they are placed on the basis of their weights on Dimensions 1 and 2 (Fig. 3) or Dimensions 3 and 4 (Fig. 4).

Item pairings, sorted by similarity With the coordinates obtained from the MDS space, it is possible to identify object pairs that are more or less similar to one another, relative to the other possible pairs. No basic unit of measurement is present in MDS, so the interitem distances are expressed in arbitrary units. This means it is not possible to define numerical cutoff values for classifying pairs as "very similar," "very dissimilar, " and so on. In order to provide empirically driven identifications of item pairs, we first created a vector of distances corresponding to the 2,016 Euclidean distances (in fourdimensional psychological space) for all item pairs. Next, the distances were rank-ordered and categorized on the basis of a quartile-split. The 504 image pairs with the closest MDS distances to one another were classified in the first quartile. The next 504 rank-ordered image pairs were classified in the
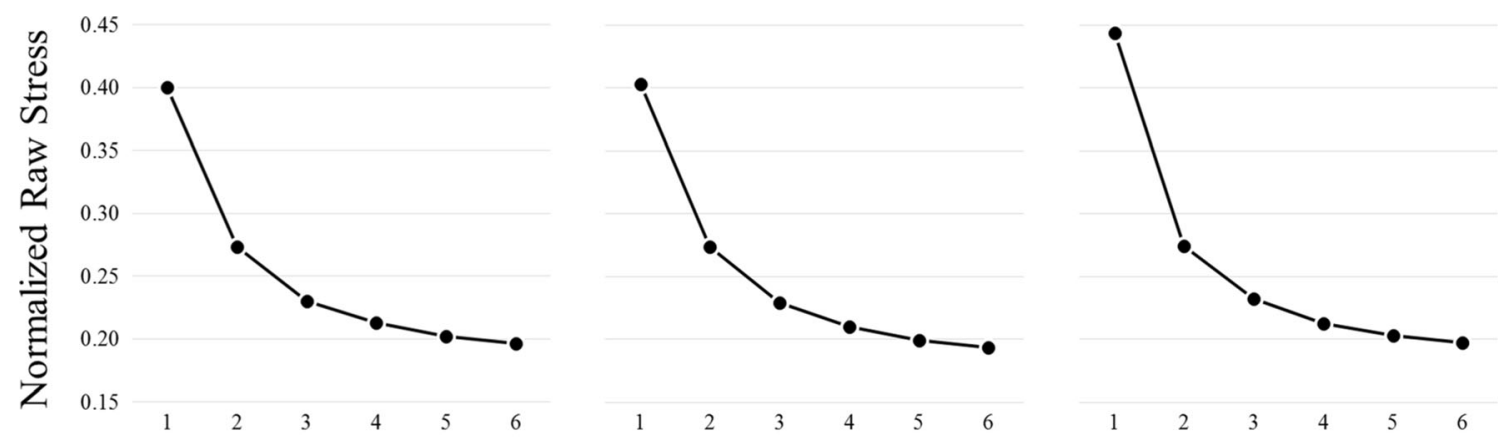

Number of Plotted Dimensions

Fig. 2 Scree plots for the Simplex (left), Torgerson (middle), and multiple-random-starts (right) options, from Experiment 2. Stress values are plotted as a function of the dimensionality in which the multidimensional scaling (MDS) data were scaled 
second quartile, and so on. For each pair of images, we provide the Euclidean distance in four-dimensional space, the ordinal ranking of the pair (where 1 is the most similar pair and 2016 is the most distal pair), and the classification of the pair (first, second, third, or fourth quartile). This classification is provided as a convenience to researchers who wish to easily identify item pairs that vary along a continuum of similarity (see Hout et al., 2014, for a similar approach). When this classification system was applied to our stimuli, we found mean MDS distances of 0.5181 for the first quartile $(S D=$ 0.1128 , range $=0.1654-0.6871), 0.7973$ for the second quartile $(S D=0.0598$, range $=0.6876-0.8877), 0.9711$ for the third quartile $(S D=0.0482$, range $=0.8879-1.0533)$, and 1.1400 for the fourth quartile $(S D=0.0641$, range $=1.0535$ $1.3593)$.

Figure 5 displays item pairings, sampled from each of the four quartiles. Shown in the top left and bottom right of the figure are the most and least similar items pairs, respectively.

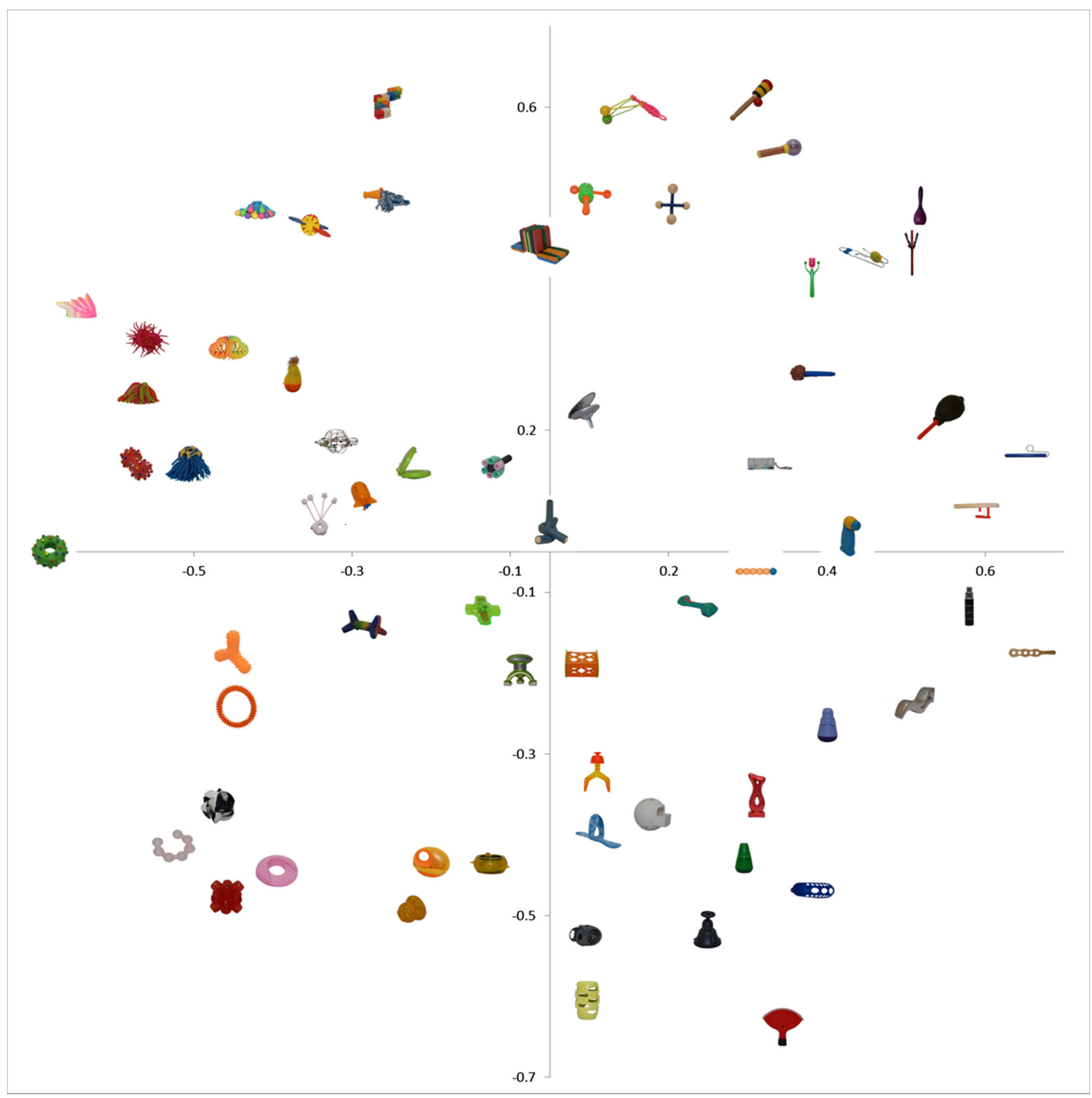

Fig. 3 Plotted results of MDS Dimensions 1 ( $x$-axis) and 2 (y-axis), with pictures superimposed, from Experiment 2 . The pictures are placed in the image on the basis of their weights on Dimensions 1 and 2 


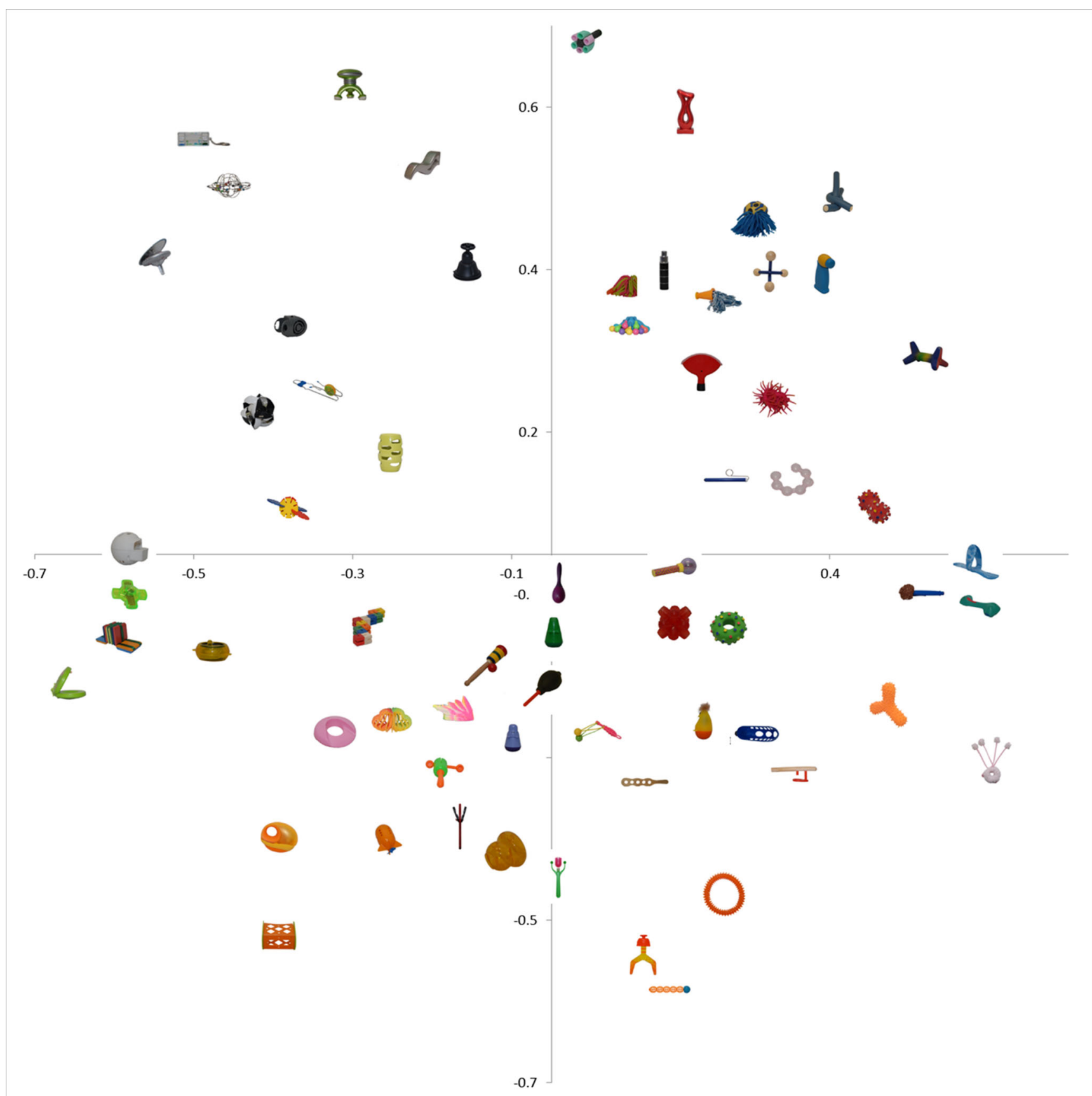

Fig. 4 Plotted results of MDS Dimensions 3 ( $x$-axis) and 4 (y-axis), with pictures superimposed (from Exp. 2). The pictures are placed in the image on the basis of their weights on Dimensions 3 and 4

The bottom left and top right of the figure show pairings sampled from the top and bottom of the second and third quartiles, respectively. It is clear that the items become less similar across quartiles. The first-quartile pair exhibit strikingly similar shapes and textures. The second- and third-quartile pairings are less alike, with similarities in overall shape for the second quartile pair, and only a vague sense of sameness for the third quartile. The fourth-quartile pair are clearly the least similar, with no discernible likenesses in shape, texture, color, and so forth.
Finally, as an aid to other researchers, we determined the 16 items that were the most and the least similar to the set as a whole. Specifically, for each object we calculated the mean of the MDS distances between that object and each of the other 63 objects. The overall grand mean was $0.8566(S D=0.0367$, range $=0.7546-0.9348)$. The 16 items with the smallest mean distances (i.e., the items most similar to the sample as a whole) and the 16 items with the largest mean distances (i.e., the items most dissimilar from the collection as a whole) are presented in Table 1. 

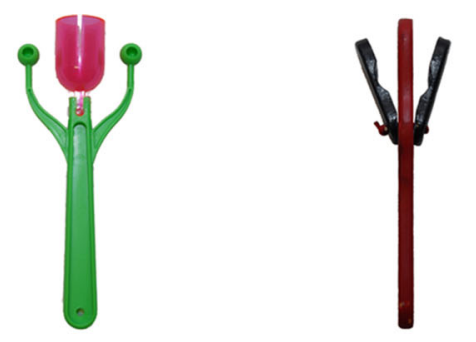

1: MDS Distance $=0.1654 ;$ Rank $=1$
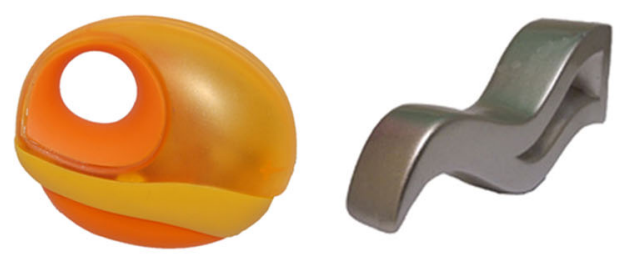

3: MDS Distance $=1.0533 ;$ Rank $=1512$

Fig. 5 Item pairs from Experiment 2, sampled from each classification quartile. The top left shows the most similar pair of items (Items 2052 and 2053), and the bottom right shows the most dissimilar pair (Items 2028

\section{Experiment 3}

In the categorization literature, there is often higher withincategory similarity for basic-level categories than for globallevel categories (see, e.g., Rosch, 1978, for a discussion). Consider, for example, the nested categories of birds, owls, and snowy owls. All birds share several features, in that they breathe, eat food, have feathers, and so forth. All owls also share features (e.g., they are predatory, they fly). Finally, all snowy owls share very specific features (e.g., yellow eyes, black beaks). At the lowest level, category members are most likely to be considered similar to one another. Moving up the hierarchy, categories become more inclusive (barn owls and great horned owls are also owls; penguins and hummingbirds are also birds), and category members become less similar as they share fewer features. Thus, our main goal with Experiment 3 was to determine whether the categories in the NOUN Database reflect this relationship between categories at different levels. In particular, we used between-category distances to determine which items, if any, belong to the same global-level categories.

\section{Method}

Participants The same participants that took part in Experiments 1 and 2 also completed Experiment 3. This experiment took place immediately after Experiment 2.

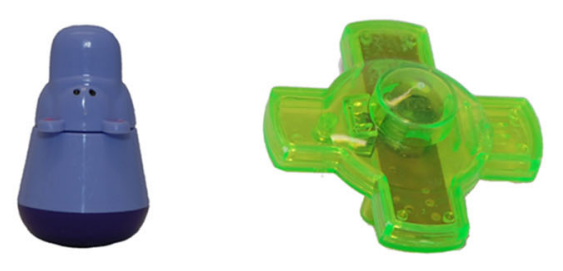

2: MDS Distance $=0.6876 ;$ Rank $=505$
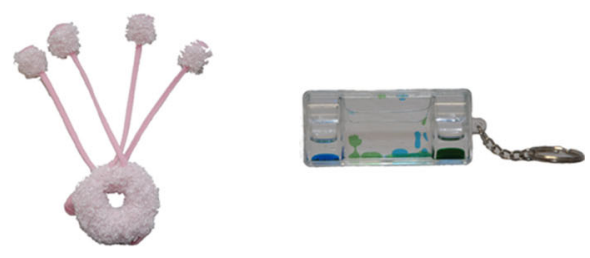

4: MDS Distance $=1.3593$; Rank $=2016$

and 2046). The top-right and bottom-left pairs are taken from the top of the second quartile and the bottom of the third quartile, respectively (top right: Items 2030 and 2039; bottom left: Items 2004 and 2031)

Stimuli Participants saw three exemplars for ten of the object categories seen in Experiments 1 and 2. We selected exemplars that only differed from each other in color, since this is a common method for forming categories for experimental research (Golinkoff, Hirsh-Pasek, Bailey, \& Wenger, 1992; Twomey et al., 2014; Vlach, Sandhofer, \& Kornell, 2008; Woodward, Markman, \& Fitzsimmons, 1994). These images were created in a fashion identical to that in Experiment 1.

Procedure and design The procedure was identical to that of Experiment 2, except that all 30 objects were displayed on the computer screen for a single SpAM trial. We included all 30 items at once so that none of the subcategories was more familiar or novel than any other category when the similarity judgments were made, and so that the overall context of the similarity judgments was the same for all participants.

\section{Results and discussion}

In order to screen our data for potential outliers, we analyzed the extent to which individual participants' MDS spaces correlated with all of the others. Because similarity is a subjective construct, it was to be expected that individual participants' solutions would deviate from those of other people. The following approach is simply a coarse measure, designed to identify participants who may not have been taking the task seriously (and therefore were only contributing noise to the data). 
Table 1 Multidimensional scaling distances for the 16 most similar and 16 least similar objects

\begin{tabular}{|c|c|c|c|}
\hline & Rank & Item & Mean Distance \\
\hline \multirow[t]{16}{*}{ Most similar items } & 1 & 2030 & 0.7546 \\
\hline & 2 & 2022 & 0.7620 \\
\hline & 3 & 2032 & 0.7721 \\
\hline & 4 & 2019 & 0.7758 \\
\hline & 5 & 2011 & 0.7956 \\
\hline & 6 & 2007 & 0.7977 \\
\hline & 7 & 2026 & 0.8104 \\
\hline & 8 & 2039 & 0.8123 \\
\hline & 9 & 2038 & 0.8131 \\
\hline & 10 & 2008 & 0.8227 \\
\hline & 11 & 2056 & 0.8295 \\
\hline & 12 & 2043 & 0.8298 \\
\hline & 13 & 2014 & 0.8307 \\
\hline & 14 & 2042 & 0.8322 \\
\hline & 15 & 2013 & 0.8344 \\
\hline & 16 & 2016 & 0.8394 \\
\hline \multirow[t]{16}{*}{ Most dissimilar items } & 49 & 2061 & 0.8781 \\
\hline & 50 & 2062 & 0.8793 \\
\hline & 51 & 2010 & 0.8798 \\
\hline & 52 & 2040 & 0.8808 \\
\hline & 53 & 2054 & 0.8811 \\
\hline & 54 & 2037 & 0.8826 \\
\hline & 55 & 2028 & 0.8871 \\
\hline & 56 & 2063 & 0.8886 \\
\hline & 57 & 2023 & 0.8923 \\
\hline & 58 & 2020 & 0.8943 \\
\hline & 59 & 2045 & 0.8984 \\
\hline & 60 & 2002 & 0.9052 \\
\hline & 61 & 2005 & 0.9095 \\
\hline & 62 & 2031 & 0.9176 \\
\hline & 63 & 2057 & 0.9307 \\
\hline & 64 & 2046 & 0.9348 \\
\hline
\end{tabular}

See the NOUN Database for images of these objects.
Conceptually, this is akin to studies of reaction time (RT) wherein participants with exceedingly long average $\mathrm{RTs}$ are removed in order to provide a cleaner estimate of the true RT.

Our approach entailed several steps (see Hout et al., 2013, for a similar approach); it should be noted that we had applied these same criteria to the data in Experiment 2, but no participants were deemed outliers in that experiment. First, we created individual MDS spaces for each participant and derived vectors of interitem distances from those spaces. Second, we correlated the distance vectors across all participants. Finally, we calculated the average correlation coefficient for each participant. We excluded one participant from further analysis for having an average correlation coefficient that was more than 2.5 standard deviations below the mean of the group.

Scree plots Scree plots for each of the starting configuration options are shown in Fig. 6. As before, the data clearly comprised more than two featural dimensions, with stress values flattening out at around four dimensions.

Configuration As before, we correlated the interitem distance vectors across solutions derived from each of the three possible starting configurations to choose the "best" configuration. Stress values were again comparable across configurations, and again, the Torgerson solution correlated most strongly with the other two (the Pearson correlation coefficients with Simplex and multiple-random-starts configurations were .93 and .89 , respectively, indicating once more a high degree of agreement across spaces). Thus, we provide the scaling solution based on the Torgerson option in four-dimensional space, with results shown in Figs. 7 and 8. In the figures, the objects are superimposed on the resulting MDS plots, such that they are placed on the basis of their weights on Dimensions 1 and 2 (Fig. 7) or Dimensions 3 and 4 (Fig. 8).

Global category classification We next compared the mean distances between the different subcategories (see
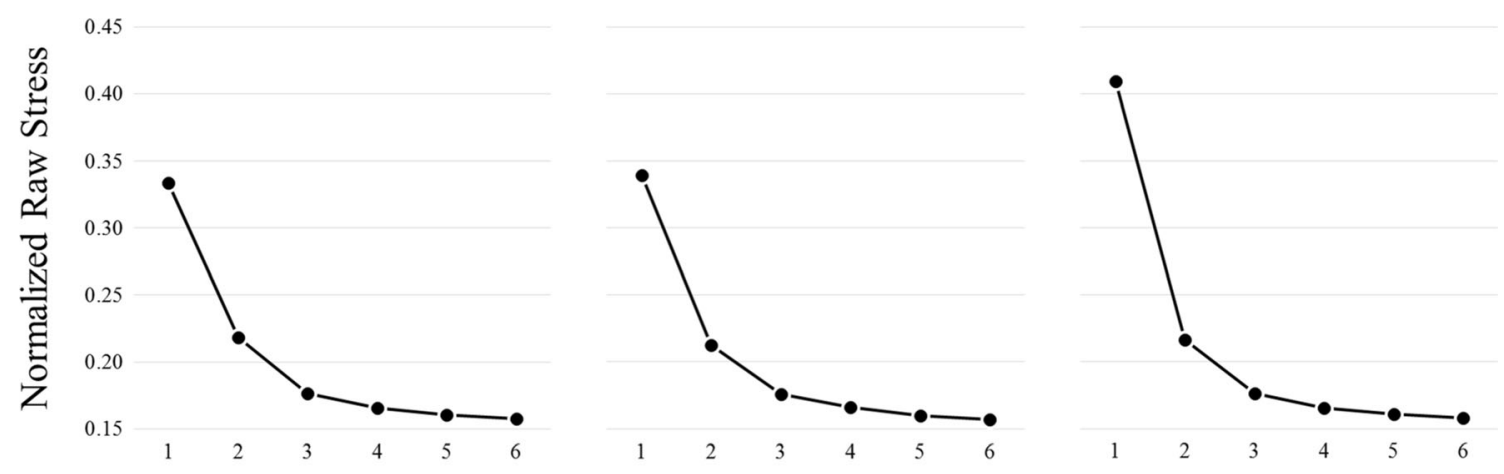

Number of Plotted Dimensions

Fig. 6 Scree plots for the Simplex (left), Torgerson (middle), and multiple-random-starts (right) options (from Exp. 3). Stress values are plotted as a function of the dimensionality in which the MDS data were scaled 
Supplemental Table 1), both within and between categories. Distances ranged from 0.06 to 1.21 in multidimensional space $\left(M_{\text {dist }}=0.78, S D_{\text {dist }}=0.37\right)$. Recall that there is no basic unit of measurement in MDS. Within-category distances were low $\left(M_{\text {dist }}=0.11, S D_{\text {dist }}=0.04\right.$, range $\left.=0.06-0.19\right)$; that is, items were considered highly similar, which we should expect, because the subcategories consisted of exemplars that only varied in their color makeup. Recall that our main goal was to use between-category distances to determine whether any items were considered to belong to the same global-level categories. Between-category distances were higher than within-category distances $\left(M_{\text {dist }}=0.93, S D_{\text {dist }}=0.20\right.$, range $\left.=0.39-1.21\right)$. We sought a relatively conservative cutoff for classifying subcategories as belonging to the same global-level category, and chose $M_{\text {dist }}+.25 S D_{\text {dist }}(=0.87)$. Using this criterion, the ten subcategories formed nine global categories (Fig. 9). Notice that three of the categories included three basic-level categories.

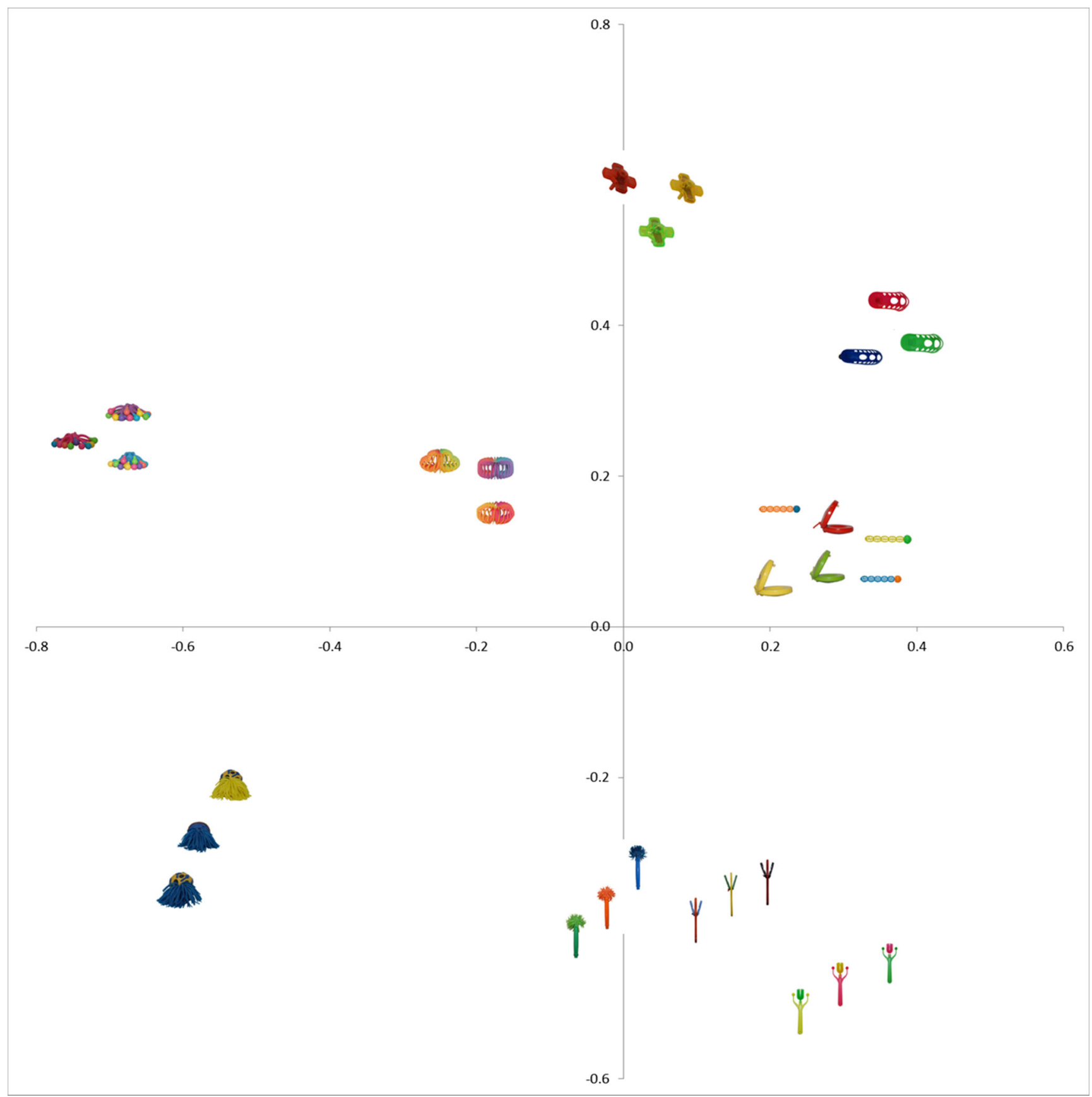

Fig. 7 Plotted results of MDS Dimensions 1 ( $x$-axis) and 2 (y-axis), with pictures superimposed (from Exp. 3). The pictures are placed in the image on the basis of their weights on Dimensions 1 and 2 
Before we continue, we will discuss the "messiness" among the global-level categories. For example, Subcategories 2015 and 2039 form a global-level (superordinate) category, and 2051 and 2039 form a global-level category, but 2015 and 2051 do not form a category. We maintain that such oddities reflect the nature of global-level categories. Consider, for example, airbus aircrafts, snowy owls, and cruise ships. One might consider airbus aircrafts and snowy owls to be members of the same global-level category, because they both fly, and airbus aircrafts and cruise ships as members of the same global-level category, because they are both large passenger transport vehicles. But one would likely not consider snowy owls and cruise ships to form a global-level category. The key point is that one's notion of similarity changes as a function of the context in which judgments are being made, and thus the relationships among object categories sometimes overlap. Thus, the "messiness" in the global-level category structure is not problematic, per

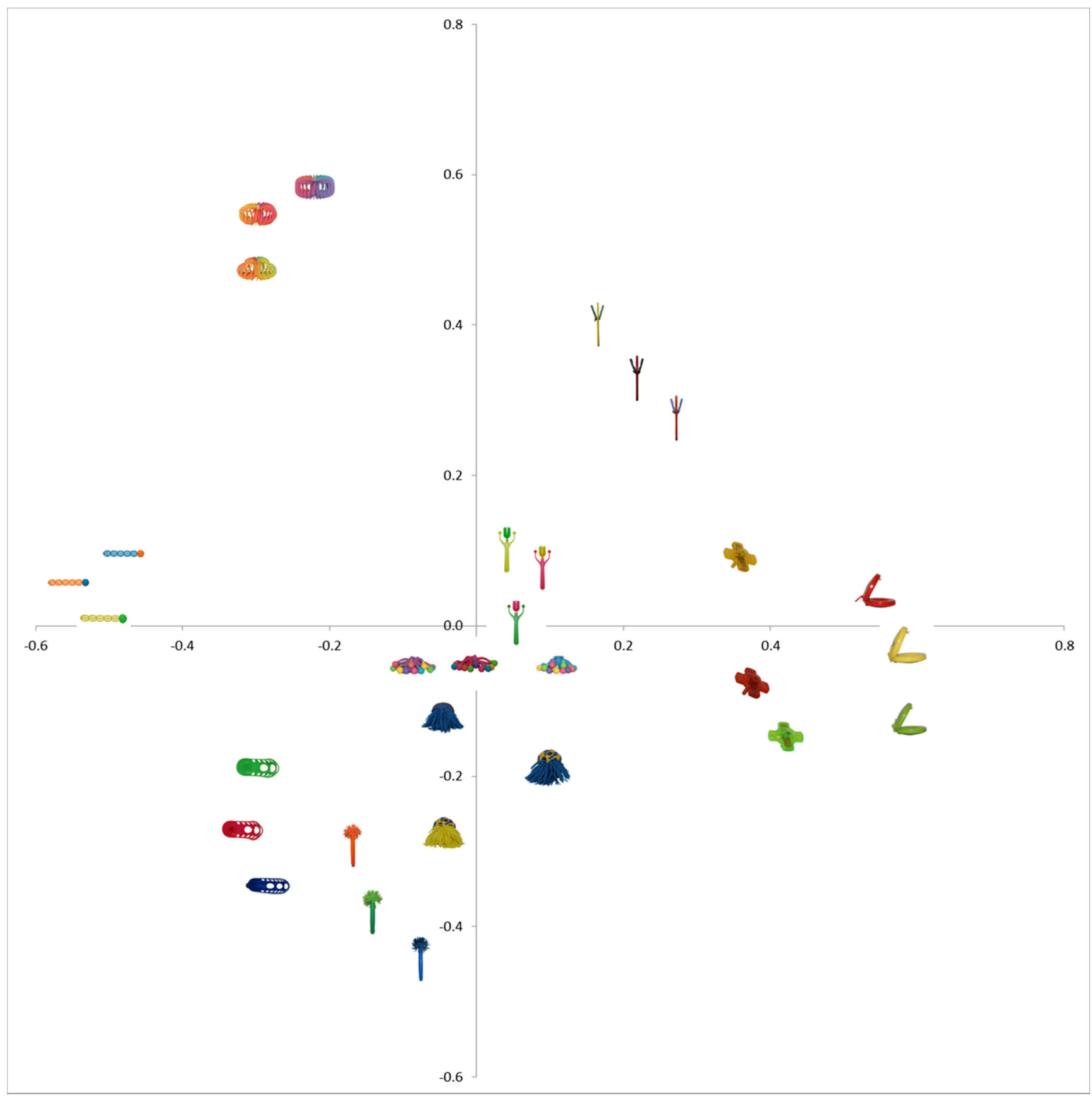

Fig. 8 Plotted results of MDS Dimensions 3 ( $x$-axis) and 4 (y-axis), with pictures superimposed (from Exp. 3). The pictures are placed in the image on the basis of their weights on Dimensions 3 and 4 
se. Rather, it is consistent with the categorization and similarity literatures (see Goldstone, Medin, \& Gentner, 1991; Hout et al., 2013). Therefore, we sought to confirm that the categories in the NOUN Database reflected this relationship between categories at different levels. To this end, we submitted the distances to a one-way ANOVA with three levels of categories: basic, global, and unrelated (e.g., 2039 and 2040). The ANOVA yielded a main effect of category, $F(2,54)=326.10, p<.001$, $\eta_{\mathrm{p}}{ }^{2}=.93$. Follow-up Tukey's HSD tests revealed greater within-category similarity for the basic-level exemplars $\left(M_{\text {basic }}=0.1150, S D_{\text {basic }}=0.0408\right.$, range $=0.0610$ $0.1894)$ than for the global-level exemplars $\left(M_{\text {global }}=\right.$ $0.7063, S D_{\text {global }}=0.1489$, range $=0.3914-0.8708, p<$ $.01)$ and than for the unrelated exemplars $\left(M_{\text {unrel }}=\right.$ $1.0517, S D_{\text {unrel }}=0.0908$, range $=0.8911-1.2144, p<$ $.01)$. The global-level exemplars also had higher withincategory similarity than the unrelated exemplars $(p<$ $.01)$. Recall that smaller numbers indicate that participants placed the items in closer spatial proximity (i.e., smaller numbers indicate greater similarity). Thus, the structure of these categories is similar to those of other categories used in experimental research.

\section{General discussion}

In this contribution, we effectively expanded the Novel Object and Unusual Name (NOUN) Database. The present series of experiments confirm that the objects included are in fact novel and can be grouped into basic- and global-level categories. Additional analyses revealed a negative relationship between novelty and consensus as to what to call the objects and what they really are. Furthermore, the more novel the objects were, the more likely participants were to qualify their responses, particularly by mentioning the colors of the objects.

The NOUN Database presents a ready-to-use collection of images of real 3-D objects. This collection of naturalistic stimuli works well alone or against images of known objects, since these objects have realistic shading, colors, textures, and complexity - often including multiple parts. The novelty ratings obtained in Experiment 1 and the similarity ratings obtained in Experiments 2 and 3 can inform researchers' decisions on which stimuli to use, depending on their research questions-for example, whether they require stimulus sets with minimal differences in novelty across items or with maximal dissimilarity between items. Thus, the use of a preexisting database, such as the NOUN Database, may help researchers

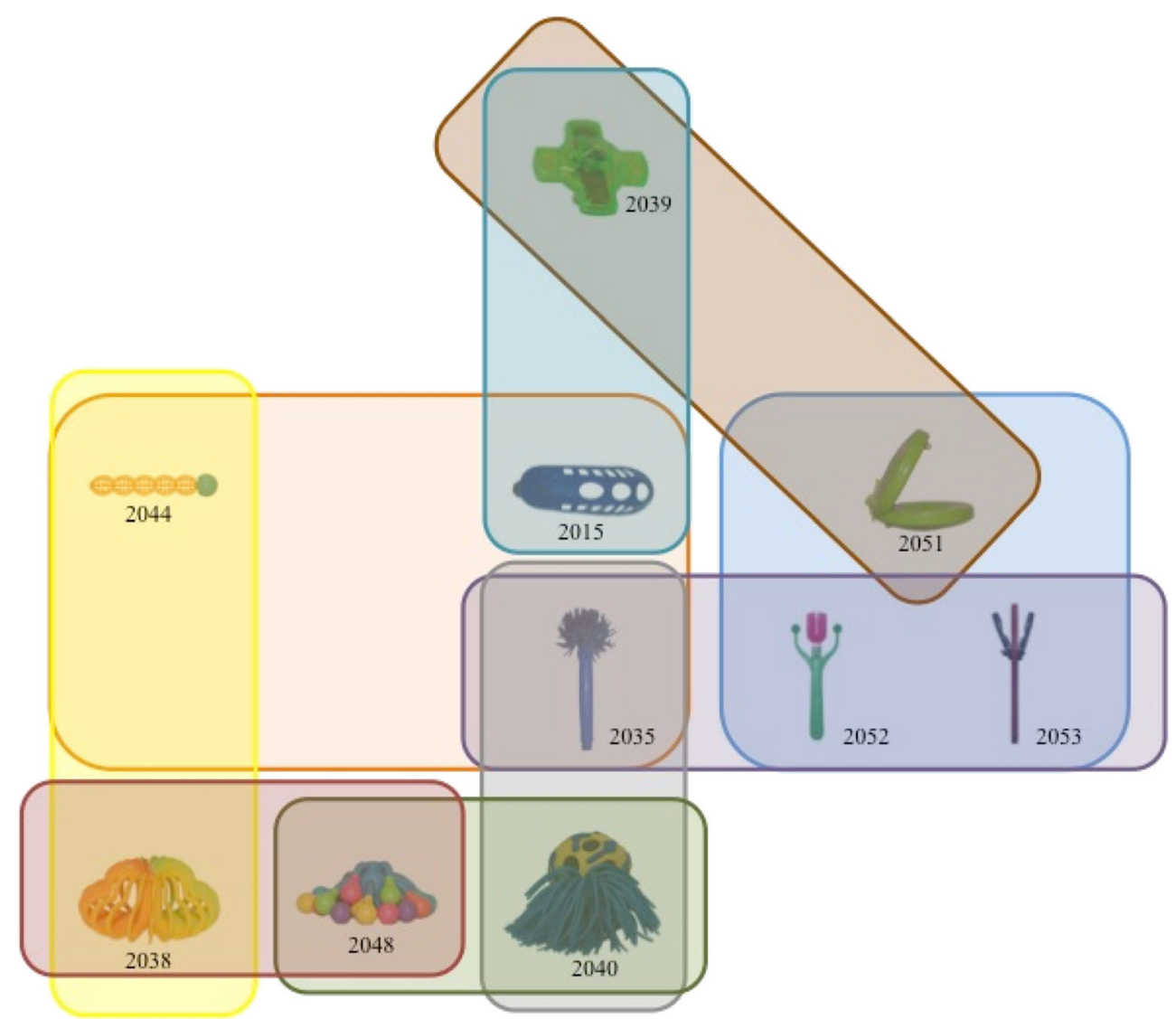

Fig. 9 Venn diagram depicting the relationships between the different categories compared in Experiment 3. Three exemplars from each category were compared; only one exemplar per subcategory is shown 
save time and cut research expenses. Importantly, the similarity ratings of multiple exemplars from ten categories (Exp. 3) provide useful information for researchers who wish to conduct experiments involving category learning and want to avoid simply relying on their own intuitive judgments of the relationships between various novel object categories.

Novel objects are useful for a variety of research designs (e.g., Axelsson \& Horst, 2013, 2014; Kwon et al., 2014), and multiple exemplars of novel objects may be useful for categorization (e.g., Homa et al., 2011; Twomey et al., 2014), memory (Hout \& Goldinger, 2010, 2012), and perception (e.g., Cunningham \& Wolfe, 2014; Hout \& Goldinger, 2015) experiments. Importantly, the objects in the NOUN Database were generally novel and were not already associated with agreedupon names. As such, these objects are particularly wellsuited for studies in which participants are learning nameobject associations for the first time (e.g., Horst et al., 2011). Specifically, the use of such novel stimuli can help ensure that any observed effects are due to the experimental manipulation and not to a priori knowledge (see also Ard \& Beverly, 2004; Bornstein \& Mash, 2010). In the real world, particularly for children, all objects were once novel, unnamed objects (Horst, 2013; Horst, McMurray, \& Samuelson, 2006) — only through cumulative, gradual learning do we learn to attribute identities to the objects around us. Therefore, using novel objects and pseudoword names is an ecologically valid design principle in a variety of settings.

The NOUN Database is a resource that will enable psychologists and empiricists in other domains to obtain the best possible experimental control in situations wherein real-world stimuli are required, but prior knowledge regarding the identity of the objects is potentially problematic. As such, this tool should allow researchers to increase the confidence in their experimental findings, and we hope it will help advance our collective scientific understanding of the processes underlying word learning, memory consolidation, categorization, and more.

Author note The creation of the original NOUN Database was funded by British Academy Small Grant Number SG-50687 to J.S.H. The expansion of the database and the preparation of this article were made possible by British Academy/Leverhulme Trust Senior Research Fellowship Number SF120032 to J.S.H. We thank Ryan Kavlie for photographing the objects and editing the photographs in the NOUN Database. We also thank Emilly Scott for her assistance with some of the materials, and Zoe Flack for help with data collection. We are indebted to Robert Goldstone for introducing the authors to each other.

\section{References}

Ard, L. M., \& Beverly, B. L. (2004). Preschool word learning during joint book reading: Effect of adult questions and comments. Communication Disorders Quarterly, 26, 17-28.
Asendorpf, J. B., Conner, M., De Fruyt, F., De Houwer, J., Denissen, J. J. A., Fiedler, K., \& Wicherts, J. M. (2013). Recommendations for increasing replicability in psychology. European Journal of Personality, 27, 108-119. doi:10.1002/per.1919

Axelsson, E. L., \& Horst, J. S. (2013). Testing a word is not a test of word learning. Acta Psychologica, 144, 264-268. doi:10.1016/j.actpsy. 2013.07.002

Axelsson, E. L., \& Horst, J. S. (2014). Contextual repetition facilitates word learning via fast mapping. Acta Psychologica, 152, 95-99. doi:10.1016/j.actpsy.2014.08.002

Berman, M. G., Hout, M. C., Kardan, O., Hunter, M., Yourganov, G., Henderson, J. M., . . Jonides, J. (2014). The perception of naturalness converges with objective measures of naturalness. PLOS ONE, 9. doi:10.1371/journal.pone. 0114572

Bion, R. A. H., Borovsky, A., \& Fernald, A. (2013). Referent selection and word learning in 18- and 24-month-old infants. Cognition, 126, 39-53. doi:10.1016/j.cognition.2012.08.008

Bornstein, M. H., \& Mash, C. (2010). Experience-based and on-line categorization of objects in early infancy. Child Development, 81 , 881-897.

Brady, T. F., Konkle, T., Alvarez, G. A., \& Oliva, A. (2008). Visual longterm memory has a massive storage capacity for object details. Proceedings of the National Academy of Sciences, 105, 1432514329. doi: 10.1073 /pnas.0803390105

Busing, F. M. R. A., Commandeur, J. J. F., Heiser, W. J., Bandilla, W., \& Faulbaum, F. (1997). PROXSCAL: A multidimensional scaling program for individual differences scaling with constraints. In W. Bandilla \& F. Faulbaum (Eds.), Advances in statistical software (Vol. 6, pp. 67-73). Stuttgart, Germany: Lucius \& Lucius.

Cunningham, C. A., \& Wolfe, J. M. (2014). The role of object categories in hybrid visual and memory search. Journal of Experimental Psychology: General, 143, 1585-1599. doi:10.1037/a0036313

Dan-Glauser, E. S., \& Scherer, K. R. V. (2011). The Geneva Affective Picture Database (GAPED): A new 730-picture database focusing on valence and normative significance. Behavior Research Methods, 43, 468-477. doi:10.3758/s13428-011-0064-1

Doyen, J., Hubaut, X., \& Vandensavel, M. (1978). Ranks of incidence matrices of Steiner triple systems. Mathematische Zeitschrift, 163, 251-260.

Ebner, N. C., Riediger, M., \& Lindenberger, U. (2010). FACES-A database of facial expressions in young, middle-aged, and older women and men: Development and validation. Behavior Research Methods, 42, 351-362. doi:10.3758/BRM.42.1.351

Gauthier, I., \& Tarr, M. J. (1997). Becoming a "greeble" expert: Exploring mechanisms for face recognition. Vision Research, 37, 1673-1682. doi:10.1016/S0042-6989(96)00286-6

Gentner, D. (1978). A study of early word meaning using artificial objects: What looks like a jiggy but acts like a zimbo. Papers and Reports on Child Language Development, 15, 1-6.

Giezen, M. R., Escudero, P., \& Baker, A. E. (in press). Rapid learning of minimally different words in five- to six-year-old children: Effects of acoustic salience and hearing impairment. Journal of Child Language. doi:10.1017/S0305000915000197

Goldstone, R. (1994). An efficient method for obtaining similarity data. Behavior Research Methods, Instruments, \& Computers, 26, 381386. doi:10.3758/BF03204653

Goldstone, R., Medin, D. L., \& Gentner, D. (1991). Relational similarity and the nonindependence of features in similarity judgments. Cognitive Psychology, 23, 222-262. doi:10.1016/0010-0285(91) 90010-L

Goldstone, R., \& Son, J. Y. (2012). Similarity. In K. J. Holyoak \& R. G. Morrison (Eds.), The Oxford handbook of thinking and reasoning (pp. 155-176). New York, NY: Oxford University Press.

Golinkoff, R. M., Hirsh-Pasek, K., Bailey, L. M., \& Wenger, N. R. (1992). Young-children and adults use lexical principles to learn 
new nouns. Developmental Psychology, 28, 99-108. doi:10.1037/ 0012-1649.28.1.99

Hahn, U. (2014). Similarity. Wiley Interdisciplinary Reviews: Cognitive Science, 5, 271-280. doi:10.1002/wcs.1282

Homa, D., Hout, M. C., Milliken, L., \& Milliken, A. M. (2011). Bogus concerns about the false prototype enhancement effect. Journal of Experimental Psychology: Learning, Memory, and Cognition, 37, 368-377. doi:10.1037/a0021803

Horst, J. S. (2009). Novel Object and Unusual Name (NOUN) Database [PDF document]. Retrieved June 6, 2014, from www.sussex.ac.uk/ wordlab/noun

Horst, J. S. (2013). Context and repetition in word learning. Frontiers in Psychology, 4(149), 1-11. doi:10.3389/fpsyg.2013.00149

Horst, J. S., McMurray, B., \& Samuelson, L. K. (2006). Online processing is essential for leaning: Understanding fast mapping and word learning in a dynamic connectionist architecture. In R. Sun (Ed.), Proceedings of the 28th Annual Conference of the Cognitive Science Society (pp. 339-344). Austin, TX: Cognitive Science Society.

Horst, J. S., \& Samuelson, L. K. (2008). Fast mapping but poor retention by 24-month-old infants. Infancy, 13, 128-157. doi:10.1080/ 15250000701795598

Horst, J. S., Samuelson, L. K., Kucker, S. C., \& McMurray, B. (2011). What's new? Children prefer novelty in referent selection. Cognition, 18, 234-244. doi:10.1016/j.cognition.2010.10.015

Horst, J. S., \& Twomey, K. E. (2013). It's taking shape: Shared object features influence novel noun generalizations. Infant and Child Development, 22, 24-43. doi:10.1002/icd.1768

Hout, M. C., \& Goldinger, S. D. (2010). Learning in repeated visual search. Attention, Perception, \& Psychophysics, 72, 1267-1282. doi:10.3758/APP.72.5.1267

Hout, M. C., \& Goldinger, S. D. (2012). Incidental learning speeds visual search by lowering response thresholds, not by improving efficiency: Evidence from eye movements. Journal of Experimental Psychology: Human Perception and Performance, 38, 90-112. doi:10.1037/a0023894

Hout, M. C., \& Goldinger, S. D. (2015). Target templates: The precision of mental representations affects attentional guidance and decisionmaking in visual search. Attention, Perception, \& Psychophysics, 77, 129-149. doi:10.3758/s13414-014-0764-6

Hout, M. C., Goldinger, S. D., \& Brady, K. J. (2014). MM-MDS: A multidimensional scaling database with similarity ratings for 240 object categories from the Massive Memory picture database. PLoS ONE, 9, e112644. doi:10.1371/journal.pone.0112644

Hout, M. C., Goldinger, S. D., \& Ferguson, R. W. (2013). The versatility of SpAM: A fast, efficient spatial method of data collection for multidimensional scaling. Journal of Experimental Psychology: General, 142, 256-281. doi:10.1037/a0028860

Hout, M. C., Papesh, M. H., \& Goldinger, S. D. (2012). Multidimensional scaling. Wiley Interdisciplinary Reviews: Cognitive Science, 4, 93 103. doi:10.1002/wcs. 1203

Imai, M., \& Gentner, D. (1997). A cross-linguistic study of early word meaning: Universal ontology and linguistic influence. Cognition, 62, 169-200. doi:10.1016/S0010-0277(96)00784-6

Jaworska, N., \& Chupetlovska-Anastasova, A. (2009). A review of multidimensional scaling (MDS) and its utility in various psychological domains. Tutorials in Quantitative Methods for Psychology, 5, 1-10.

Konkle, T., Brady, T. F., Alvarez, G. A., \& Oliva, A. (2010). Conceptual distinctiveness supports detailed visual long-term memory for realworld objects. Journal of Experimental Psychology: General, 139, 558-578. doi:10.1037/a0019165

Kriegeskorte, N., \& Mur, M. (2012). Inverse MDS: Inferring dissimilarity structure from multiple item arrangements. Frontiers in Psychology, 3, 245. doi:10.3389/fpsyg.2012.00245

Kucker, S. C., \& Samuelson, L. K. (2012). The first slow step: Differential effects of object and word-form familiarization on retention of fast-mapped words. Infancy, 17, 295-323. doi:10. 1111/j.1532-7078.2011.00081.x

Kwon, M.-K., Luck, S. J., \& Oakes, L. M. (2014). Visual short-term memory for complex objects in 6- and 8-month-old infants. Child Development, 85, 564-577. doi:10.1111/cdev.12161

Landau, B., Smith, L. B., \& Jones, S. (1998). Object shape, object function, and object name. Journal of Memory and Language, 38, 1-27.

Lipinski, J., \& Gupta, P. (2005). Does neighborhood density influence repetition latency for nonwords? Separating the effects of density and duration. Journal of Memory and Language, 52, 171-192. doi: 10.1016/j.jml.2004.10.004

Marchewka, A., Żurawski, Ł., Jednoróg, K., \& Grabowska, A. (2014). The Nencki Affective Picture System (NAPS): Introduction to a novel, standardized, wide-range, high-quality, realistic picture database. Behavior Research Methods, 46, 596-610. doi:10.3758/ s13428-013-0379-1

Mather, E., \& Plunkett, K. (2009). Learning words over time: The role of stimulus repetition in mutual exclusivity. Infancy, 14, 60-76.

Mather, E., Schafer, G., \& Houston-Price, C. (2011). The impact of novel labels on visual processing during infancy. British Journal of Developmental Psychology, 29, 783-805. doi:10.1348/2044-835X. 002008

Matheson, H. E., \& McMullen, P. A. (2011). A computer-generated face database with ratings on realism, masculinity, race, and stereotypy. Behavior Research Methods, 43, 224-228. doi:10.3758/s13428010-0029-9

Migo, E. M., Montaldi, D., \& Mayes, A. R. (2013). A visual object stimulus database with standardized similarity information. Behavior Research Methods, 45, 344-354. doi:10.3758/s13428012-0255-4

Navarro, D. J., \& Griffiths, T. L. (2008). Latent features in similarity judgments: A nonparametric Bayesian approach. Neural Computation, 20, 2597-2628. doi:10.1162/neco.2008.04-07-504

Navarro, D. J., \& Lee, M. D. (2003). Combining dimensions and features in similarity-based representations. In S. Becker, S. Thrun, \& K. Obermayer (Eds.), Advances in Neural Information Processing Systems (pp. 59-66). Cambridge, MA: MIT Press.

Navarro, D. J., \& Lee, M. D. (2004). Common and distinctive features in stimulus similarity: A modified version of the contrast model. Psychonomic Bulletin \& Review, 11, 961-974. doi:10.3758/ BF03196728

Nosofsky, R. M. (1986). Attention, similarity, and the identification-categorization relationship. Journal of Experimental Psychology: General, 115, 39-57. doi:10.1037/0096-3445.115.1.39

Rakison, D. H., \& Poulin-Dubois, D. (2002). You go this way and I'll go that way: Developmental changes in infants' detection of correlations among static and dynamic features in motion events. Child Development, 73, 682-699. doi:10.1111/1467-8624.00432

Rosch, E. (1978). Principles of categorization. In E. Rosch \& B. B. Lloyd (Eds.), Cognition and categorization (pp. 27-48). Hillsdale, NJ: Erlbaum.

Rost, G. C., \& McMurray, B. (2009). Speaker variability augments phonological processing in early word learning. Developmental Science, $12,339-349$

Samuelson, L. K., \& Horst, J. S. (2007). Dynamic noun generalization: Moment-to-moment interactions shape children's naming biases. Infancy, 11, 97-110. doi:10.1207/s15327078in1101_5

Samuelson, L. K., \& Smith, L. B. (1999). Early noun vocabularies: Do ontology, category structure and syntax correspond? Cognition, 73, 1-33. doi:10.1016/S0010-0277(99)00034-7

Samuelson, L. K., \& Smith, L. B. (2005). They call it like they see it: Spontaneous naming and attention to shape. Developmental Science, 8, 182-198. doi:10.1111/j.1467-7687.2005.00405.x

Schneider, W., Eschman, A., \& Zuccolotto, A. (2002). E-prime computer software and manual. Pittsburgh, PA: Psychology Software Tools, Inc. 
Shepard, R. N. (1980). Multidimensional scaling, tree-fitting, and clustering. Science, 210, 390-398. doi:10.1126/science.210.4468.390

Shepard, R. N. (1987). Toward a universal law of generalization for psychological science. Science, 237, 1317-1323. doi:10.1126/ science. 3629243

Shepard, R. N., \& Arabie, P. (1979). Additive clustering: Representation of similarities as combinations of discrete overlapping properties. Psychological Review, 86, 87-123. doi:10.1037/0033-295X.86.2.87

Smith, A. K., Ayanian, J. Z., Covinsky, K. E., Landon, B. E., McCarthy, E. P., Wee, C. C., \& Steinman, M. A. (2011). Conducting high-value secondary dataset analysis: An introductory guide and resources. Journal of General Internal Medicine, 26, 920-929. doi:10.1007/ s11606-010-1621-5

Smith, J. D., \& Minda, J. P. (2002). Distinguishing prototype-based and exemplar-based processes in dot-pattern category learning. Journal of Experimental Psychology: Learning, Memory, and Cognition, 28, 800-811. doi:10.1037/0278-7393.28.4.800

Smith, L. B., \& Yu, C. (2008). Infants rapidly learn word-referent mappings via cross-situational statistics. Cognition, 106, 1558-1568. doi:10.1016/j.cognition.2007.06.010

Soja, N. N., Carey, S., \& Spelke, E. S. (1992). Perception, ontology, and word meaning. Cognition, 45, 101-107. doi:10.1016/00100277(92)90025-D

Tversky, A. (1977). Features of similarity. Psychological Review, 84, 327-352. doi:10.1037/0033-295X.84.4.327

Twomey, K. E., Ranson, S. L., \& Horst, J. S. (2014). That's more like it: Multiple exemplars facilitate word learning. Infant and Child Development, 23, 105-122. doi:10.1002/icd.1824

Umla-Runge, K., Zimmer, H. D., Fu, X., \& Wang, L. (2012). An action video clip database rated for familiarity in China and Germany.
Behavior Research Methods, 44, 946-953. doi:10.3758/s13428012-0189-x

Vitevitch, M. S., \& Luce, P. A. (1998). When words compete: Levels of processing in perception of spoken words. Psychological Science, 9, 325-329. doi:10.1111/1467-9280.00064

Vlach, H. A., Sandhofer, C. M., \& Kornell, N. (2008). The spacing effect in children's memory and category induction. Cognition, 109, 163167. doi:10.1016/j.cognition.2008.07.013

Warren, D. E., \& Duff, M. C. (2014). Not so fast: Hippocampal amnesia slows word learning despite successful fast mapping. Hippocampus, 24, 920-933. doi:10.1002/hipo.22279

Werker, J. F., Cohen, L. B., Lloyd, V. L., Casasola, M., \& Stager, C. L. (1998). Acquisition of word-object associations by 14-month-old infants. Developmental Psychology, 34, 1289-1309. doi:10.1037/ 0012-1649.34.6.1289

Woodward, A. L., Markman, E. M., \& Fitzsimmons, C. M. (1994). Rapid word learning in 13- and 18-month-olds. Developmental Psychology, 30, 553-566. doi:10.1037/0012-1649.30.4.553

Wu, R., Gopnik, A., Richardson, D. C., \& Kirkham, N. Z. (2011). Infants learn about objects from statistics and people. Developmental Psychology, 47, 1220-1229. doi:10.1037/a0024023

Yu, C., \& Smith, L. B. (2007). Rapid word learning under uncertainty via cross-situational statistics. Psychological Science, 18, 414-420. doi: 10.1111/j.1467-9280.2007.01915.x

Zosh, J. M., Brinster, M., \& Halberda, J. (2013). Optimal contrast: Competition between two referents improves word learning. Applied Developmental Science, 17, 20-28. doi:10.1080/ 10888691.2013.748420 